\title{
A 5G Vehicle-to-Everything Ecosystem with Internet of Vehicles based Approaches
}

\section{Carlos R. Storck, Fátima Duarte-Figueiredo (Supervisor)}

\author{
${ }^{1}$ Pontifical Catholic University of Minas Gerais - Graduate Program in Informatics \\ ${ }^{2}$ Federal Center for Technological Education of Minas Gerais (CEFET-MG) \\ storckecefetmg.br, fatimafig@pucminas.br
}

\begin{abstract}
The Fifth Generation (5G) cellular network is a good infrastructure option to connect vehicles through the Cellular-based Vehicle-to-Everything $(C-V 2 X)$ technology. $C-V 2 X$ aims to provide drivers comfort, road safety, autonomous steering for reliable connections and Advanced Driver Assistance Systems (ADAS). To $5 G$ effectively provide $C$-V2X communications, there are many questions to answer and many problems to solve. One of them is the absence of an architecture that absorbs and characterizes the vehicles as special things in the Internet. The connectivity continuity guarantees after a handover is a huge problem because the vehicles have high mobility. It raises their handover occurrences as well as failed and unnecessary handovers. The thesis titled "A $5 G$ Vehicle-to-Everything Ecosystem with Internet of Vehicles based Approaches" goals were the characterization of the IoV (Internet of Vehicles) architecture supported by a $5 G$ network on $C$-V2X communications, the proposition of a $5 G \mathrm{~V} 2 \mathrm{X}$ ecosystem, the presentation of an user-centered Virtual Cells (V-Cells) selection probabilistic approach, a vehicular handover decision solution based on complex network metrics for $5 G$ Ultra-Dense Network (UDN) and the validation of a structured model as a new $5 G$ service provider facilitator. This thesis proposal and contributions include FiVH (5G Vehicular Handover) a solution that does the virtual cells selection and conformation and deals with the frequent handovers to provide many IoV services, such as on board video entertainment applications. The $C$-V2X applications can be efficiently supported by the $5 G$ vehicle-to-everything ecosystem IoV communications.
\end{abstract}

\section{Contextualization, Problem Characterization and Motivation}

The past century's future vision used to include people talking through screens, smart vehicles, everything interconnected with everything. The future has arrived. The vehicles are among many things connected to the Internet. They are increasingly intelligent, automatic, and connected. The vehicular technology can be useful in the accidents minimization, monitoring and controlling traffic. They can also provide multimedia applications entertainment on board. Vehicles can be connected among themselves in Vehicle-to-Vehicle (V2V) mode, or they can be connected to other elements in a virtual environment called Vehicle-to-Everything (V2X). The Internet of Vehicles (IoV) is an open and integrated network system with several components, including vehicles, people, and things that need a good infrastructure to be part of smart cities.

The Fifth Generation (5G) cellular network is the certainly infrastructure to connect vehicles. The virtual environment created by the Cellular-based V2X (C-V2X) 
technology can provide greater comfort to drivers, safety roads and autonomous steering for more reliable connections. The V2X application types are divided into two basic operations: Device-to-Device (D2D) and Vehicle-to-Network (V2N). The IoV solutions must be compatible with the $5 \mathrm{G}$ network and beyond. The $5 \mathrm{G}$ ultra-high bandwidth through millimeter Wave (mmWave) frequencies and the wireless technology innovations are some features to provide C-V2X. Vehicles, things, and people fully connected are the main goal of the V2X and IoV technologies. The cellular high density causes difficulties in the handover cell selection, mainly for vehicles that have high mobility and speed. Thus, without a huge control, there may be a great number of failed and unnecessary handovers (ping-pong effect), more packet losses, longer delays and high energy consumption. The vehicular mobility also makes it difficult to guarantee QoS, maintaining the nodes connection with the provided services. One of the most important problems in this field is the communication continuity guarantee. Frequent handovers due to vehicular high mobility and speed are listed as some of the major problems around this field. Complex vehicular multimedia applications need coverage and quality of service (QoS) guarantees. An integrated infrastructure ecosystem must take care of these complex aspects.

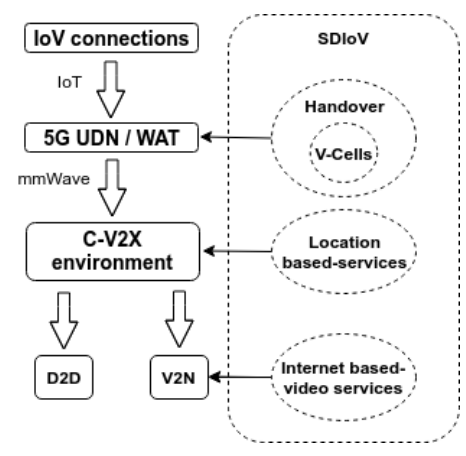

Figure 1. C-V2X ecosystem context.

\section{Goals}

This thesis proposes IoV connection approaches over a 5G V2X ecosystem illustrated by the scheme showed in the Figure 1. The ecosystem has incorporated the Software Defined Internet of Vehicles (SDIoV) concept. As specific goals, we can cite:

- IoV architecture characterization and the 5G C-V2X ecosystem proposition;

- A probabilistic user-centered Virtual Cell (V-Cell) selection based on complex network metrics;

- Indication of a handover solution for 5G Ultra-Dense Network (UDN) connected vehicles;

- Validation of a 5G network technology structured model as a new service provider facilitator.

\section{Related Work}

Some related works are shown in this section. They are part of our published IEEE Access survey [Storck and Duarte-Figueiredo 2020c]. Several 5G researches are taking place in many countries. Some of them are about the International Mobile Telecommunications for 2020 (IMT-2020) and beyond, targeting: radio regulations, operational aspects, 
protocols, Quality of Service (QoS) and Experience (QoE); the 5G specifications, targeting: RAN, service and systems aspects; the 5G technologies, targeting: mmWave transmission, 5G protocols, MEC and NFV; the 5G initiative, targeting: technology evolution towards 5G; the 5G Public-Private Partnership (5G-PPP) projects, targeting: 5G infrastructure; the technical community, targeting: 5G development; the 5G network development on Americas, targeting: promotion for the full development of wireless technology capabilities; and much more [Storck and Duarte-Figueiredo 2019a]. 5G is going to develop the best technological solution to enable V2X improvements to provide V2V, V2P, V2I, V2N communications [Festag 2015, Chen et al. 2017]. To evaluate the V2X implementation, the 5G-DRIVE is a study-project that is going on in the European Union (EU) and in China to harmonize researches and trials [Chen 2019]. The usercentered virtual cells consist of several transmission points with an association pattern for each user and his/her respective mobility [Sahin et al. 2017, Sahin et al. 2018]. The virtual cells are formed by the association of a user with several Transmission Points (TPs) around him/her. The V-Cell moves because of the user. For this, a controller is required to dynamically configure and manage virtual cells, which can be instantiated by network slice according to specific service requirements. Sahin et al. [Sahin et al. 2018] present a V-Cell concept, that can be applied in several V2X use cases where broadcast communications. The authors assumed that through co-operative beamforming all the TPs in a V-Cell are transmitting the same data in parallel to a vehicle so there is no intraVC (V-Cell) interference. However, inter-V interference needs to be considered as well as an user-centered approach, since a vehicular group is very unstable and difficult to control due to the vehicles high mobility and speed.

\section{Results}

The Figure 2 shows the proposed 5G V2X ecosystem. It has as differences to other literature architectures: a connection between the virtual Baseband Unit (BBU) polls and an SDN controller. It communicates through the backhaul with the core network $(\mathrm{CN}$ $5 \mathrm{GC}$ ) with the separation of the control plane from the data plane. At the $\mathrm{CN}$, the Serving Gateway (S-GW) and Packet data network Gateway (P-GW) elements are unified as User Plane Function (UPF). Servers such as the V2X-Server can be enabled in the Central Office Data Center to support new services.

The proposed ecosystem includes approaches to the virtual cell installation, the deal with the frequent handovers, and location-based services under Software-Defined Networking (SDN) control to enable an efficient performance of C-V2X communication in applications supported by the 5G network through IoV connections. The ecosystem selects three potential virtual cells based on vehicle-centric probabilistic approach, using complex network metrics, before the handover itself. The FiVH (5G Vehicular Handover) mechanism makes the handover decision based on proper criteria. The developed procedure is shown in Fig. 3. It represents the operating cycle of the handover operation. Each vehicle is linked to a set of antennas on the Base Stations (BSs) that make up virtually a V-Cell. A V-Cell is created and managed by NFV. The used multicriteria differentiate $\mathrm{FiVH}$ from the solutions proposed in the literature and provide performance gains in the handover, decrease in the handover time, decrease in the number of unnecessary (ping-pong effect), decrease in the number of failed handovers, and also decrease of lost packets. 
Figure 2. 5G V2X ecosystem

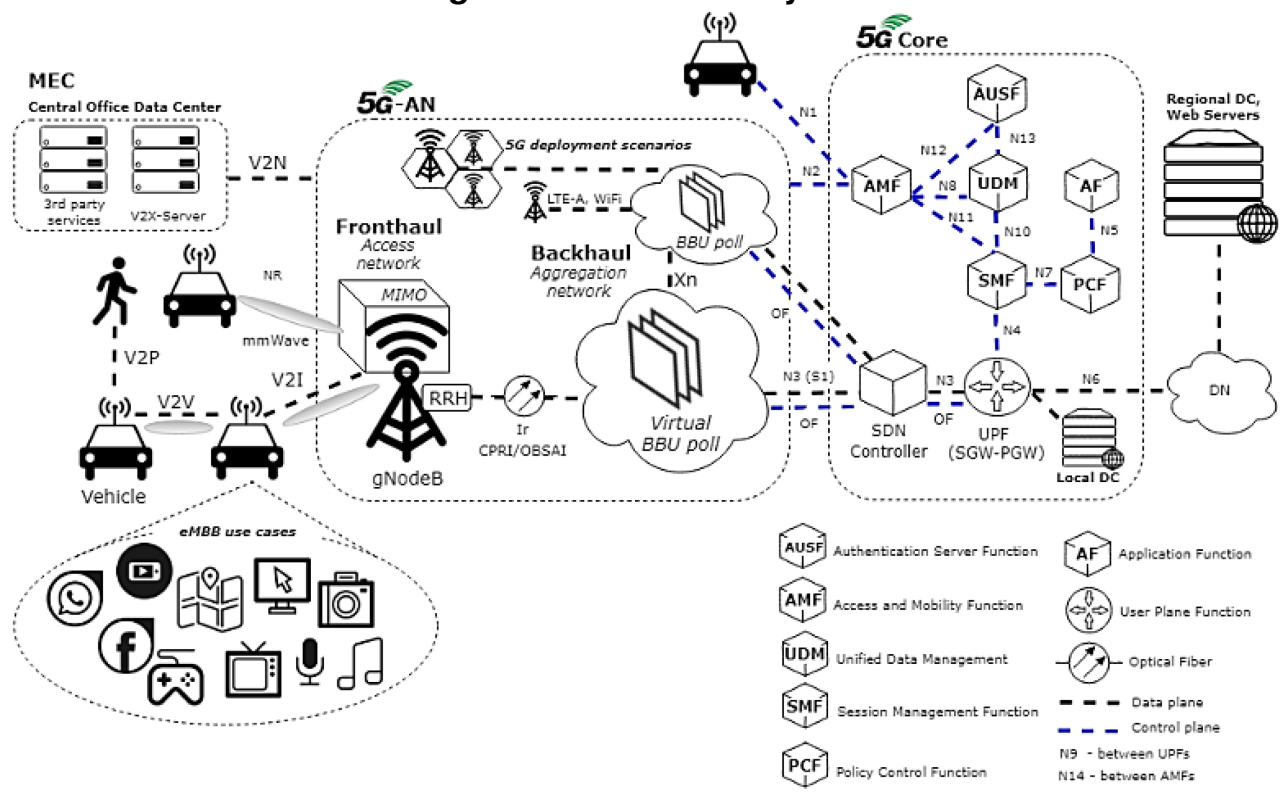

Figure 3. Handover procedure of the FiVH

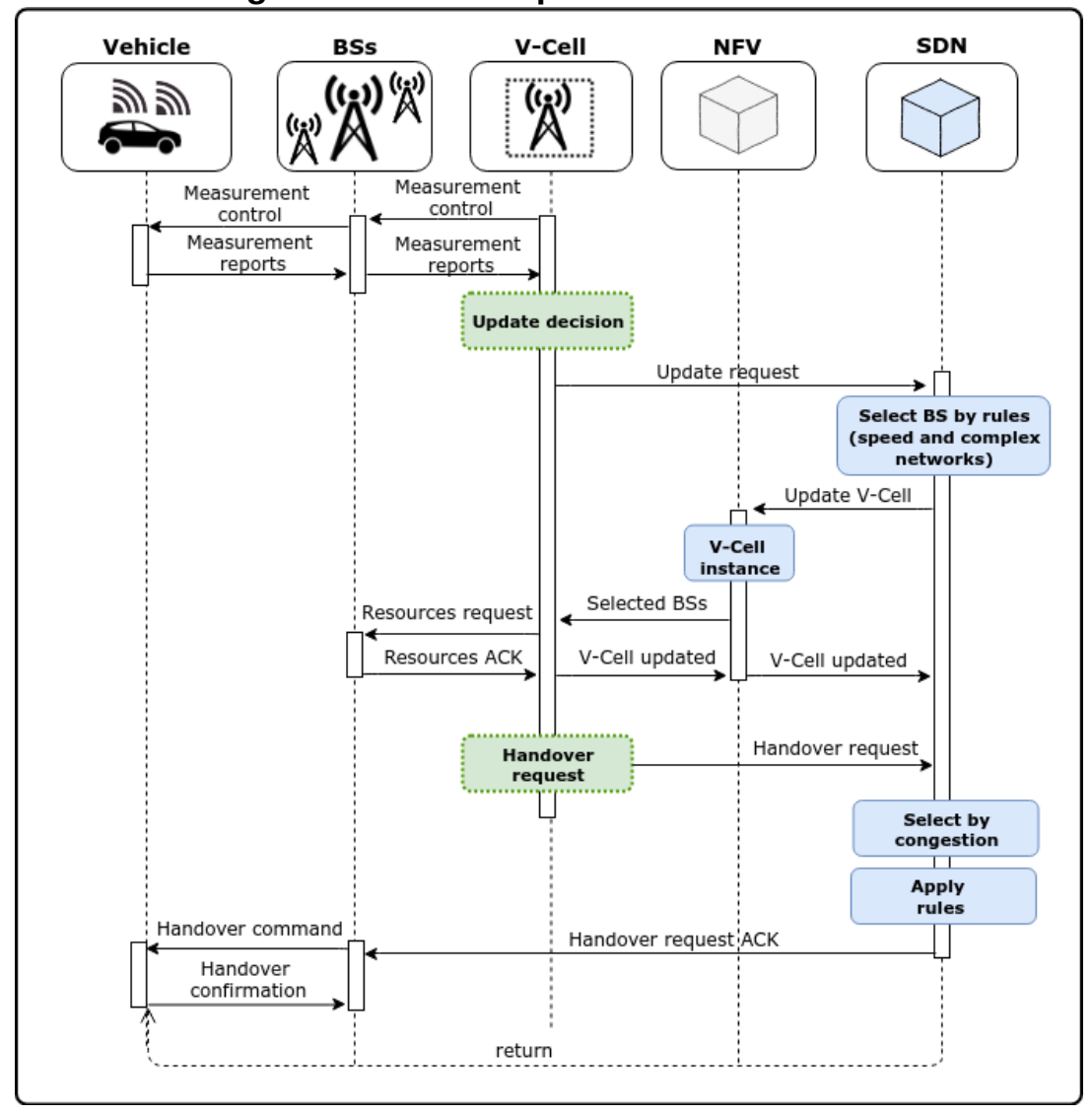

As the thesis has too many results, graphics, tables and simulations, it follows a summary of its five parts. In the first part (chapter 2), the IoV V2X architecture is presented. In the second part (chapter 3 ), the $5 \mathrm{G} \mathrm{V} 2 \mathrm{X}$ ecosystem is proposed. A validation 
of it was conducted through simulations with a Dynamic Adaptive Streaming over HTTP (DASH) as an adaptive algorithm used in rural and urban scenarios with different densities of vehicle connected to the $5 \mathrm{G}$. Some results considering playback interruptions, video quality, transitions, buffer level, bit-rate and frequency shown the ecosystem effectiveness. In the third part (chapter 4), a vehicle-centric probabilistic approach is presented to address the problem of V-Cells management in an UDN with scenarios of vehicles in high mobility. The results show the successfully use of complex networks metrics to the cell selection. In the fourth part (chapter 5), the main contribution of the thesis is finally presented: the $5 \mathrm{G}$ Vehicular Handover $(\mathrm{FiVH})$ as a handover decision solution for vehicles that are connected in 5G UDN. It has used the inter-v-cell selection method previous described. To compose the decision criterias, the FiVH used also a congestion control and a network capacity balance proposal, the average vehicular speed and standardized Quality of Service (QoS) rules. It made a combination of complex network metrics to choose the best virtual cell for a vehicle in motion through an optimal path. The FiVH solution considered the integration with a SDN controller, since the $5 \mathrm{G} \mathrm{C-V2X}$ needs high-speed mobility support. In the fifth part (chapter 6), a 5G model, named Urban Computing Framework in 5G Networks (CoUrbF5G), was proposed for location-based services to support V2X applications and other smart city services. The ns-3 discrete event simulator and the Mininet network emulator were used for simulations and emulations. The obtained results have shown the efficiency of the proposed framework to guarantee quality of services using $5 \mathrm{G} \mathrm{V} 2 \mathrm{X}$ communications.

As an example of the thesis many results, Figure 4 graphic shows the V2V packet delivery results. It is possible to observe, that the delivery rate depends on vehicle density. In the literature, traditional VANETs present the PDR of between $60-80 \%$. By simulations, when adopting $5 \mathrm{G}$ millimeter waves to transmit the messages, considering the confidence interval of $95 \%$, a constant behavior is observed, between $84 \%$ and $91 \%$ of vehicles that received the package, in all vehicle densities. Generally, the delivery rate tends to be low in scenarios with low vehicle density. However, it is noted that the obtained result is due to the adoption of $5 \mathrm{G}$ ecosystem in a cell of mist, evidencing the approach efficiency to provide IoV.

Figure 4. Packet delivery ratio in 5G V2V communications

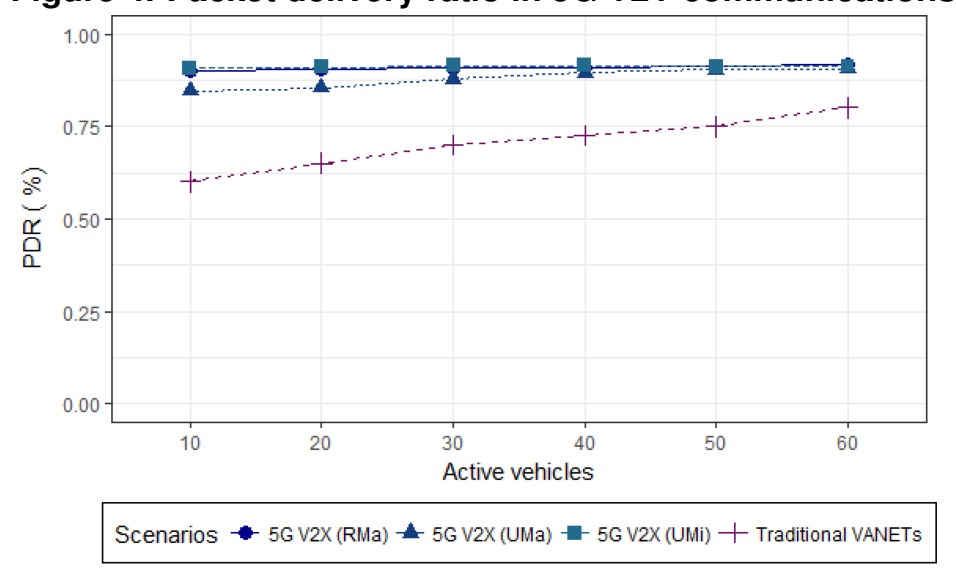

As another example of the thesis many results, Figure 5 represents the percentage of FiVH failed HOs by the percentage of HOs performed over time. According to 
Gharsallah et al. [Gharsallah et al. 2018], the average handover delay is close to 200 $\mathrm{ms}$ (in $4 \mathrm{G}$ ), and the rate of failed handovers is higher when compared to FiVH.

Figure 5. Handover failure ratio

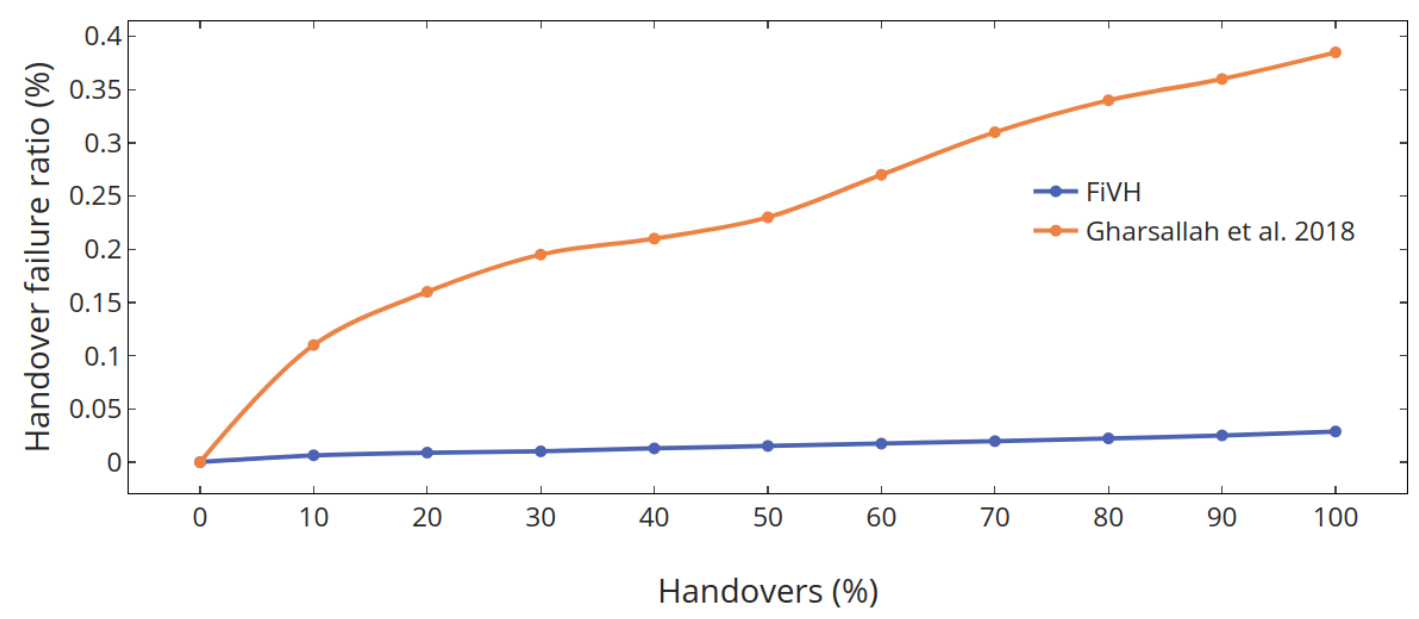

\section{Contributions and Publications}

The thesis contributions derived from each chapter are listed in Table 1. Twelve published papers ( 5 journals and 7 conferences) in renowned publication vehicles were derived from the thesis. The grade shown in the table is based in the 2021 CAPES Qualis predicted score (A1 is the highest one, corresponding to a high JCR grade).

\section{Conclusions and future research directions}

It is expected that network-connected vehicles will behave like cell phones in a very near future. This is a relevant aspect for the proposal of new network ecosystems and models. The IoV still represents a challenge. A C-V2X architecture involves multiple communication technologies that can be used to support fully vehicular applications and services. There is an urgent need of solutions proposed in this direction, like this thesis 5G V2X ecosystem for IoV. The proposed ecosystem includes the CoUrbF5G framework, a vehicle-centric probabilistic approach to address the problem of V-Cells management, with the use of complex networks metrics to the cell selection, and the the 5G Vehicular Handover $(\mathrm{FiVH})$ as a handover decision solution for vehicles that are connected in $5 \mathrm{G}$ UDN. Each part of this $5 \mathrm{G}$ ecosystem can guarantee vehicular connectivity, availability and quality of service, as shown in the detailed thesis results and publications.

The $5 \mathrm{G}$ is promoting a great business change by enabling new services and improving the existing ones. The technology enablers in RAT areas for V2X communications and network virtualization affect business relationships. They create more collaborative business environments to find solutions for the increasingly industrial and commercial demands in a new universe of possibilities. International standards, protocols and security concerns about the network connectivity, the user privacy, and autonomous vehicles effective control require future work. Future works shall bring new tests in $5 \mathrm{G}$ scenarios with the development of real services applied to smart cities. 
Table 1. Author's contributions and publications

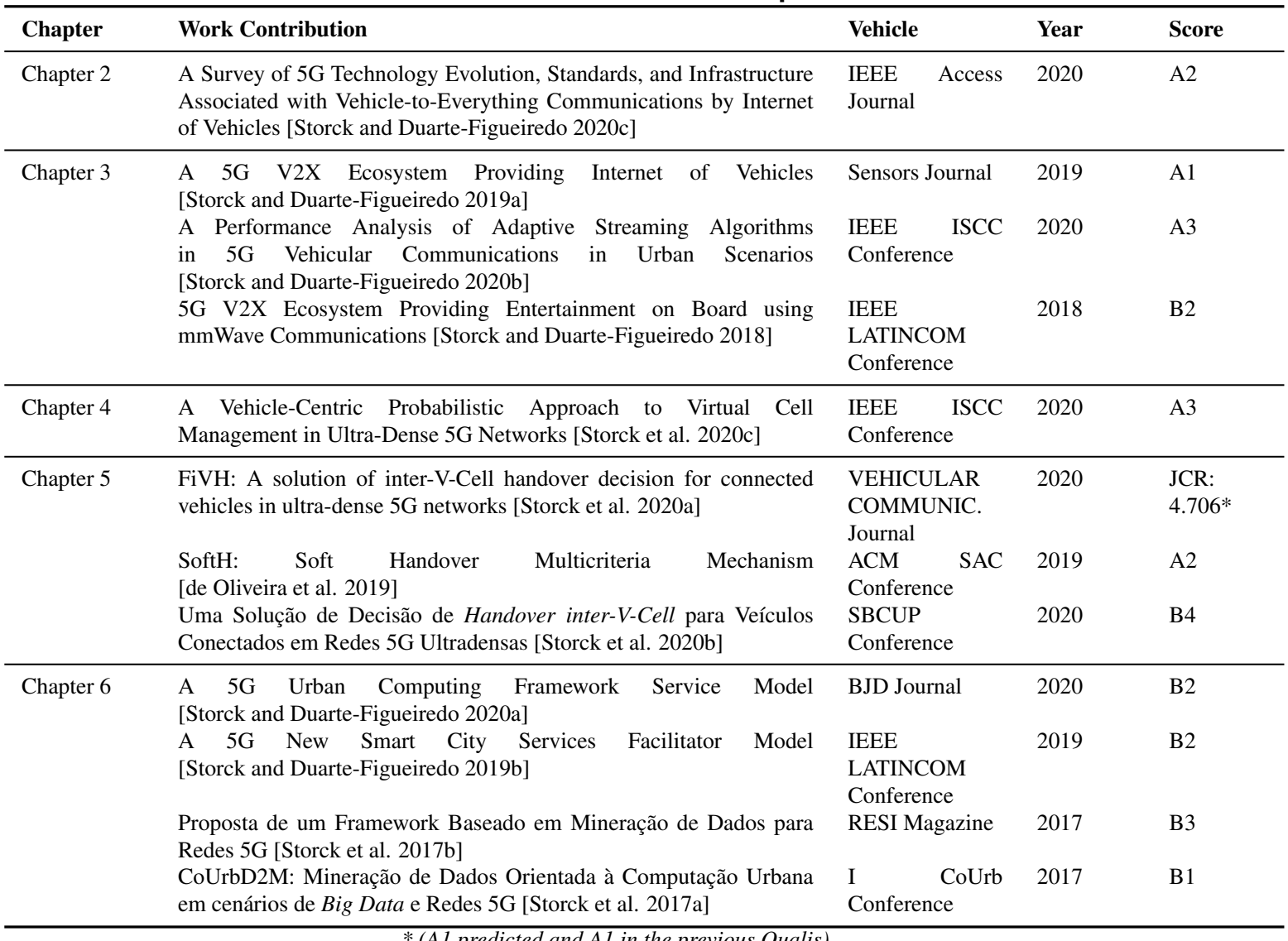

\section{Acknowledgments}

This thesis was financed in part by the Coordination of Higher Level Personnel Improvement (CAPES) - Brazil - Finance Code 001. This work was supported in part by the CEFET-MG and in part by the PUC Minas.

\section{References}

Chen, S., Hu, J., Shi, Y., Peng, Y., Fang, J., Zhao, R., and Zhao, L. (2017). Vehicleto-Everything ( $2 \mathrm{x}$ ) Services Supported by LTE-Based Systems and 5G. IEEE Communications Standards Magazine, 1(2):70-76.

Chen, T. (2019). C-V2X Trial Activities in 5G-DRIVE. In Workshop on $5 G$ in the Era of Connected Cars, pages 1-12, Valencia, Spain. EuCNC.

de Oliveira, G. R. M., Storck, C. R., and Duarte-Figueiredo, F. (2019). SoftH: soft handover multicriteria mechanism. In Proc. of the 34th ACM/SIGAPP Symposium on Applied Computing (SAC '19), pages 2422-2429, Limassol, Cyprus. ACM.

Festag, A. (2015). Standards for vehicular communication - from IEEE 802.11p to 5G. $e$ \& i Elektrotechnik und Informationstechnik, 132(7):409-416.

Gharsallah, A., Zarai, F., and Neji, M. (2018). SDN/NFV-based handover management approach for ultradense 5G mobile networks. International Journal of Communication Systems, page e3831. 
Sahin, T., Klugel, M., Zhou, C., and Kellerer, W. (2018). Virtual Cells for 5G V2X Communications. IEEE Communications Standards Magazine, 2(1):22-28.

Sahin, T., Klügel, M., Zhou, C., and Kellerer, W. (2017). Multi-user-centric virtual cell operation for V2X communications in 5G networks. In 2017 IEEE Conference on Standards for Communications and Networking (CSCN), pages 84-90, Helsinki, Finland. IEEE.

Storck, C. R., de O. Lousada, E. E., de O. Silva, G. G., Mini, R. A., and DuarteFigueiredo, F. (2020a). FiVH: A solution of inter-V-Cell handover decision for connected vehicles in ultra-dense 5G networks. Vehicular Communications, pages $1-13$.

Storck, C. R., de O. Lousada, E. E., de O. Silva, G. G., Mini, R. A., and DuarteFigueiredo, F. (2020b). Uma Solução de Decisão de Handover inter-V-Cell para Veículos Conectados em Redes 5G Ultradensas. In 12th Brazilian Symposium on Ubiquitous and Pervasive Computing (SBCUP), pages 1-10, Cuiabá, Brazil. SBC.

Storck, C. R. and Duarte-Figueiredo, F. (2018). 5G V2X Ecosystem Providing Entertainment on Board Using MmWave Communications. In 2018 IEEE 10th LatinAmerican Conference on Communications (LATINCOM), pages 1-6, Guadalajara, Mexico. IEEE.

Storck, C. R. and Duarte-Figueiredo, F. (2019a). A 5G V2X Ecosystem Providing Internet of Vehicles. Sensors, 19(3).

Storck, C. R. and Duarte-Figueiredo, F. (2019b). A 5G New Smart City Services Facilitator Model. In 2019 IEEE 11 th Latin-American Conference on Communications (LATINCOM), pages 1-6, Salvador, Brazil. IEEE.

Storck, C. R. and Duarte-Figueiredo, F. (2020a). A 5G Urban Computing Framework Service Model. Brazilian Journal of Development, 6(6):1-16.

Storck, C. R. and Duarte-Figueiredo, F. (2020b). A Performance Analysis of Adaptive Streaming Algorithms in 5G Vehicular Communications in Urban Scenarios. In 25th IEEE Symposium on Computers and Communications (ISCC), pages 1-7, Rennes, France. IEEE.

Storck, C. R. and Duarte-Figueiredo, F. (2020c). A Survey of 5G Technology Evolution, Standards, and Infrastructure Associated with Vehicle-to-Everything Communications by Internet of Vehicles. IEEE Access, 8:117593-117614.

Storck, C. R., Sales, E., Zarate, L. E., and Duarte-Figueiredo, F. (2017a). CoUrbD2M: Mineração de Dados Orientada à Computação Urbana em cenários de Big Data e Redes 5G. In 1st Workshop on Urban Computing (CoUrb) - 35th Brazilian Symposium on Computer Networks and Distributed Systems (SBRC), pages 1-6, Belém, Brazil. SBC.

Storck, C. R., Sales, E., Zarate, L. E., and Duarte-Figueiredo, F. (2017b). Proposta de um Framework Baseado em Mineração de Dados para Redes 5G. RESI, 16(2):1-16.

Storck, C. R., Silva, G. G. O., and Duarte-Figueiredo, F. (2020c). A Vehicle-Centric Probabilistic Approach to Virtual Cell Management in Ultra-Dense 5G Networks. In 25th IEEE Symposium on Computers and Communications (ISCC), pages 1-7, Rennes, France. IEEE. 